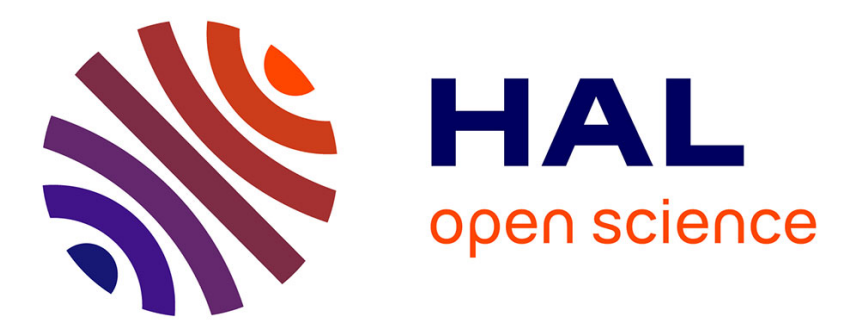

\title{
The role of interaction in virtual embodiment: Effects of the virtual hand representation
}

\author{
Ferran Argelaguet Sanz, Ludovic Hoyet, Michaël Trico, Anatole Lécuyer
}

\section{To cite this version:}

Ferran Argelaguet Sanz, Ludovic Hoyet, Michaël Trico, Anatole Lécuyer. The role of interaction in virtual embodiment: Effects of the virtual hand representation. IEEE Virtual Reality, Mar 2016, Greenville, United States. pp.3-10, 10.1109/VR.2016.7504682 . hal-01346229

\section{HAL Id: hal-01346229 \\ https://inria.hal.science/hal-01346229}

Submitted on 18 Jul 2016

HAL is a multi-disciplinary open access archive for the deposit and dissemination of scientific research documents, whether they are published or not. The documents may come from teaching and research institutions in France or abroad, or from public or private research centers.
L'archive ouverte pluridisciplinaire HAL, est destinée au dépôt et à la diffusion de documents scientifiques de niveau recherche, publiés ou non, émanant des établissements d'enseignement et de recherche français ou étrangers, des laboratoires publics ou privés.

\section{(c)(1)}

Distributed under a Creative Commons Attribution| 4.0 International License 


\title{
The Role of Interaction in Virtual Embodiment: Effects of the Virtual Hand Representation
}

\author{
Ferran Argelaguet* \\ Inria Rennes
}

\author{
Ludovic Hoyet ${ }^{\dagger}$ \\ Inria Rennes
}
Michaël Trico
UPMC
Anatole Lécuyer $\$$
Inria Rennes
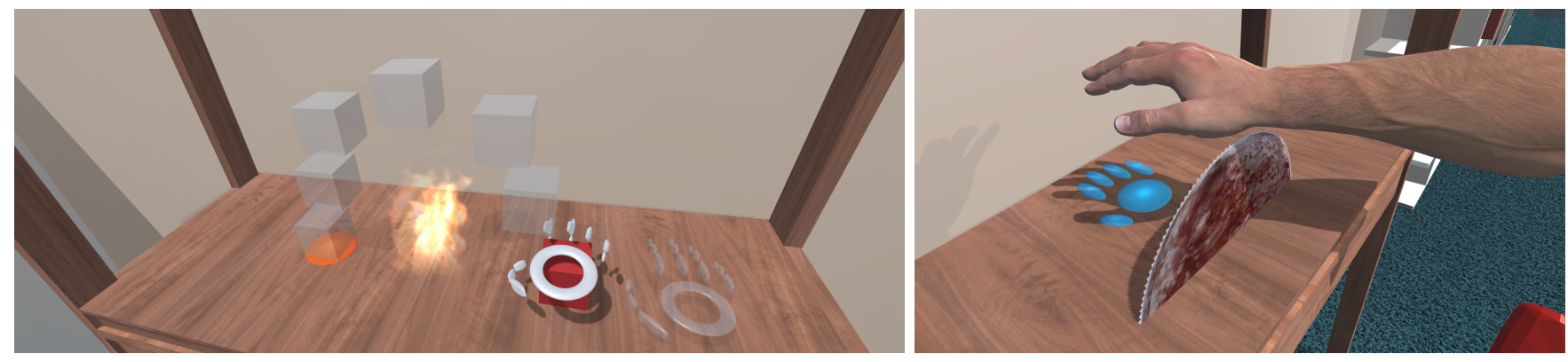

Figure 1: The presented experiment assessed the sense of embodiment when interacting with virtual hands with different levels of realism. (Left) Participants performed a series of pick-and-place tasks avoiding different obstacles (the iconic virtual hand and the "fire" obstacle are depicted). (Right) Additionally, participants performed a task in which the virtual hand was potentially threatened by a spinning saw.

\begin{abstract}
How do people appropriate their virtual hand representation when interacting in virtual environments? In order to answer this question, we conducted an experiment studying the sense of embodiment when interacting with three different virtual hand representations, each one providing a different degree of visual realism but keeping the same control mechanism. The main experimental task was a Pick-and-Place task in which participants had to grasp a virtual cube and place it to an indicated position while avoiding an obstacle (brick, barbed wire or fire). An additional task was considered in which participants had to perform a potentially dangerous operation towards their virtual hand: place their virtual hand close to a virtual spinning saw. Both qualitative measures and questionnaire data were gathered in order to assess the sense of agency and ownership towards each virtual hand. Results show that the sense of agency is stronger for less realistic virtual hands which also provide less mismatch between the participant's actions and the animation of the virtual hand. In contrast, the sense of ownership is increased for the human virtual hand which provides a direct mapping between the degrees of freedom of the real and virtual hand.
\end{abstract}

Index Terms: H.5.2 [Information Interfaces and Presentation]: User Interfaces-Evaluation/Methodology I.3.7 [Computer Graphics]: Three-Dimensional Graphics and Realism-Virtual Reality;

\section{INTRODUCTION}

The virtual representation of the user in immersive virtual environments, the avatar, has elicited a lot of attention both in virtual reality and psychological research communities [11]. Does the user perceive the avatar as her/his own body? Is the avatar able to al-

\footnotetext{
*e-mail:fernando.argelaguet_sanz@inria.fr

†e-mail:ludovic.hoyet@inria.fr

$\ddagger$ e-mail:michael.trico@laposte.net

$\S$ e-mail:anatole.lecuyer@inria.fr
}

ter the user's self perception and/or behavior? Virtual reality is a powerful tool to answer these questions as the user's avatar can be altered in numerous ways in order to assess changes in the sense of embodiment and behavior [15]. For example, controlling the level of realism of the avatar [23], its skin color [22] or even its shape [26]. However, little is known about the role of interaction towards the sense of embodiment. Existing works mainly focused on the effects of visuo-tactile [30], visuo-motor stimuli [24] or morphological changes [38].

In this work, we explore the effects of different virtual hand representations on the sense of embodiment when actively interacting with the virtual environment (see Figure 1). We aim to grow the existing knowledge on how the representation of the user alters the perception of the virtual environment, the avatar and her/his self, in which performance and appreciation are not necessarily correlated [28]. We designed an experiment in which participants performed a series of pick-and-place operations in which, sometimes, hazardous elements could threaten their virtual hand (see Figure 1). Three different visual representations of the virtual hand were considered, each providing different degrees of realism (shape and grasping animations). However, all of them shared the same underlying grasping control scheme, providing the same interaction capabilities. The study focused on two dimensions of the sense of embodiment: the sense of agency, i.e. the feeling of being in control of the avatar, and the sense of ownership, i.e. the feeling that the avatar is the source of experienced sensations. Two main research questions are addressed: Does the virtual representation of the hand alter the sense of agency? and, Does the virtual representation of the hand alter the sense of ownership? The obtained results show that the sense of agency is related to the virtual hand control and the task efficiency, while the sense of ownership is mainly related to the visual appearance of the virtual hand. These results can help the design of interactive systems focusing on virtual embodiment.

The remainder of the paper is structured as follows. Section 2 provides a broad overview of related work on virtual grasping, embodiment and existing body ownership illusions in VR. Section 3 presents the methodological basis of the experiment and details the analysis of the experimental results. Then, Section 4 discusses the main findings and Section 5 provides the concluding remarks. 


\section{BACKGROUND}

\subsection{Virtual Grasping}

Grasping is one of the most common interactions performed in everyday life. Still, the simulation of realistic grasping operations in virtual environments requires dedicated input devices and algorithmic approaches [4]. In this work we are only focusing on egocentric interaction techniques not requiring physically-based simulations. Egocentric manipulation techniques, such as the virtual hand [27], translate the user's hand movements to a simplified virtual representation of the hand, in which objects are typically glued to the virtual hand upon contact. Virtual hand metaphors can be enhanced by providing an increased control of the virtual hand (e.g. finger motions [17]) and providing additional visual feedback [32]. Regarding the hand control, finger displacement and orientation can be used as an heuristic to determine the fingers configuration to infer grasping operations [36, 21]. Furthermore, in order to provide additional feedback, the visual representation of the hand or of the interactive objects can be altered in order to express contacts, valid grasping status [21] or use explicit glyphs and illumination effects [32].

\subsection{The Sense of Embodiment}

In this Section we will briefly detail the different elements considered to be the main actors to enable the sense of embodiment. For additional reading we will refer to the works of Kilteni et al. [15] and De Vignemont [8].

Kilteni et al. [15] defines the sense of embodiment ( $\mathrm{SoE}$ ) toward a body $B$ as the sense that emerges when B's properties are processed as if they were the properties of one's own biological body. Embodiment is a complex phenomena which is achieved at different levels, as defined by Longo et al. [19] and further revisited by Kilteni et al. [15]: the phenomenology of embodiment includes the sense of self-location, the sense of agency, and the sense of body ownership. A similar decomposition was provided by De Vignemont [8] in which three dimensions are considered: Spatial, Motor and Affective. While spatial and motor dimensions are directly related to self-location and of agency respectively, the sense of ownership is linked to the affective dimension. A stronger sense of ownership will increase the physical and physiological responses towards hazardous situations that threaten the virtual body.

\subsubsection{Self-location}

Self-location can be defined as the space in which we perceive the self to be located. The body space provides a reference frame for our physical body and determines the space in which body sensations are registered [8]. Several factors can alter the sense of self-location. A collocation between the virtual and the real body (first person perspective) will elicit a stronger sense of self-location that non-collocated perspectives (third person perspective) $[31,25]$. In addition, synchronous visuo-proprioceptive correlations during passive or active movements increase the sense of self-location. The well known rubber hand illusion experiment [5] showed that self-location can be altered when synchronous visuo-proprioceptive correlations are applied between the rubber hand and the hidden real hand. Furthermore, correlated vestibular cues can also increase the sense of location [2].

\subsubsection{Agency}

The sense of agency is elicited when oneself is the agent of one's own actions. When interacting with our body, we have accurate control of the motor activity and we are aware of our actions (e.g. proprioception). Agency is described as motor activity control, which encompasses the obedience of the concerned body part to one's will and the sensation of movement [2]. In other words, agency is closely related to action awareness and action planning [8].
The sense of agency is present in the use of tools (effectors), for which the knowledge of sensorimotor control and the association between effectors leads to an expected outcome. The close relationship between intention and outcome is also considered as a component of agency [6]. When controlling virtual limbs or fullbody avatars, the sense of agency has little impact on the effectors. For example, when controlling virtual avatars, the sense of agency appears even when the avatars are not realistic (such as point-line avatars [37]), when virtual avatars drastically deviate from the physical body [16] or with virtual limbs in implausible positions [35]. Although motor recalibration is required when the effector (e.g. tool, virtual body) differs with respect to the real body, a degree of visuomotor adaptation is tolerated in forms of proprioceptive recalibration, motor learning or virtual space recalibration [7]. Nevertheless, the perceptual-motor fidelity between individuals and their avatars must be ensured.

\subsubsection{Ownership}

The sense of ownership is described as the sense that one's own body is the source of sensations [35]. Since Botvinik and Cohen's experiment with a rubber hand [5], known as the rubber hand illusion (RHI), it has been proved that a fake limb can elicit the sense of ownership. While the brain can believe that a fake limb belongs to the body $[34,13]$, a basic morphological similarity or spatial configuration between the real and artificial body is still required [15].

Ownership can be observed when the fake body is threatened [8]. For example, on the RHI, the fact of hitting the rubber hand created a strong physical response [5]. Although one might argue that the response is related to the surprise effect, neuroscience studies proved that the reaction induced by the threatening of the rubber hand (which is to retract one's own hand in the vast majority of cases) is not only a pure reflex, but also that there is a cortical anxiety response to a perceived danger towards the body [1].

\subsection{Body Ownership Illusions in VR}

Going one step further, the RHI has been revisited in VR, giving birth to the virtual arm illusion [30]. Similar to the RHI, stimulations between the avatar and the real hand should be synchronous. However, studies performed in VR setups [24] show that the virtual arm illusion can be achieved without tactile stimuli. More precisely, Slater et al. [29] show that synchrony between visual and proprioceptive information along with motor activity is able to induce an illusion of ownership over a virtual arm. Additional studies have explored full-body ownership, some examples of illusions have used mannequins [23], virtual avatars [31,3] or even out-ofbody experiences [18].

Such experiments paved the way for further studies to explore how changes in the virtual avatar influence the sense of ownership. For instance, body space and limb plausibility have been investigated in [16], in which participants tolerated having a virtual arm longer than their real one. Another example is the work of Peck et al. [22], which showed that racial bias could be reduced by using an avatar of a black person. Additional studies have explored one's body weight perception by altering the complexity of the avatar [26], adding additional limbs to the avatar [9, 33] or even exploring the effects of social anxiety responses to standing in front of an audience when having an invisible body [12].

Although there is evidence that the virtual representation of the user has an impact on the sense of ownership [12, 22, 26], few studies have explored how the virtual body and its interaction capabilities alter the user's behavior. Existing studies have focused on constrained interactions with the virtual environment, limited to pushing virtual buttons [10], touching landmarks [20] or drumming [14]. The following experiment goes one step further and evaluates the effects of the avatar representation in a realistic setup where believable interactions with the environment are provided. 


\section{EXPERIMENT}

How do people appropriate the virtual representation of their hand when interacting with virtual environments? The following experiment aims to study the effect of the hand representation towards the sense of agency and ownership. The two research questions studied were (1) does the representation of the virtual hand influence the sense of agency? and (2) does the representation of the virtual hand influence the sense of ownership?

In order to address both research questions, we designed an experiment in which the user had to perform a set of pick-and-place tasks. The main factor of the experiment was the representation of the virtual hand, from an iconic virtual hand to a fully animated one (see Figure 2). The task was designed so that participants have to actively interact with their virtual hand. Additionally, we designed a second task in which participants were asked to place their virtual hand in a predefined virtual location in which they could potentially put their virtual hand in danger. The sense of ownership and agency were assessed by analyzing the participant's behavior when interacting with the virtual environment and by gathering their subjective impressions. We first detail the virtual grasping technique used, followed by the experimental tasks and methods, and the analysis and discussion of the results.

\subsection{Virtual Grasping Techniques}

\subsubsection{Virtual Hand Representation}

The design of the virtual hands took the degree of realism into consideration. As previously discussed, the degree of morphological similarity could have an impact on the sense of ownership, and the control is tightly coupled with the sense of agency. Three different virtual hand representations were considered, each one providing a different level of realism (see Figure 2). All virtual hands were collocated with the real hand.

Abstract virtual hand. Low realism. The hand is represented by a uniformly shaded sphere which moves according to the real hand palm. Once the grasping operation is triggered, the shading color of the sphere changes from white to red. Most VR applications still use such a representation.

Iconic virtual hand. Medium realism. The 3D model represents a simplified robotic hand. A two state animation is played (opened to closed, there is no continuous animation) when the grasping operation is triggered. The hand model is translated and rotated following the real hand palm position and orientation.

Realistic virtual hand. High realism. This representation is a fully animated virtual hand (including the forearm) which follows the user's arm, hand and finger movements. The 3D model was directly obtained from the Leap Motion SDK.

\subsubsection{Grasping Control}

Although having different visual representations, all three virtual hand representations shared the same grasping control scheme. The prehension area was considered to be a half-sphere (with the same radius as the abstract virtual hand) driven by the palm of the real hand. In order to interact with an object, the prehension area had to collide with it. Once the prehension area collides with the object, users had to close/open their real hand in order to trigger the grasping/release operation.

Hand tracking was provided by the Leap Motion (see Figure 4), allowing to track the forearm, the hand and the fingers of the user's dominant hand. We decided to use the Leap Motion because it provided a seamless finger tracking without the need to wear gloves or markers, thus providing less invasive tracking. The tracking quality ensured a correct animation for the realistic virtual hand for most situations, still, when the palm was in a vertical position finger tracking issues appeared due to inter-finger occlusions. To avoid such situations, finger animation was deactivated when the palm was in a vertical position. This situation rarely occurred during the
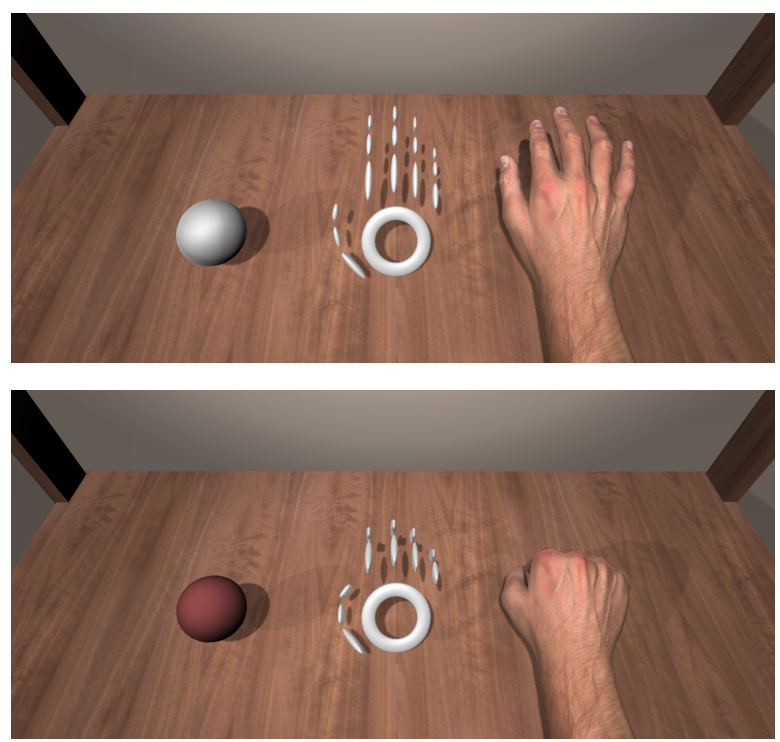

Figure 2: Virtual hand representations. Abstract (left), iconic (center) and realistic virtual hands (right). Each virtual hand had its own visual feedback when the grasping operation is triggered (bottom). The abstract virtual hand changes color, the iconic virtual hand abruptly changes shape (there is no smooth animation) and the realistic virtual hand is animated from the user's finger motions.

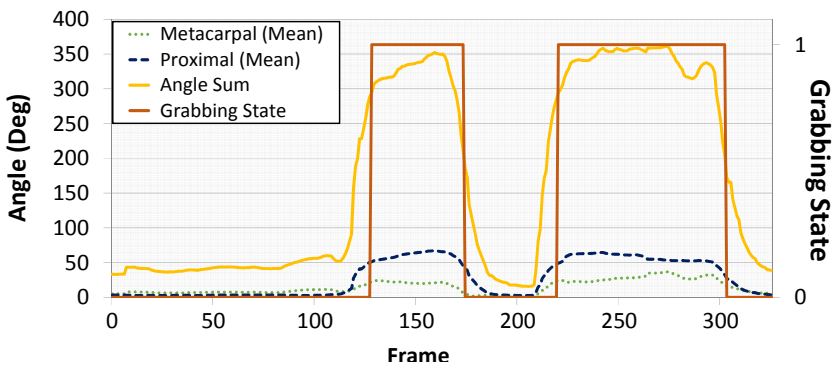

Figure 3: Dashed lines show the mean flexion values for the metacarpal and proximal joints during two consecutive grasping operations. In order to control the grasping state, the sum of the angle of all metacarpal and proximal joints was used. The grasping and release thresholds were set at 290 and 200 degrees.

experimental tasks as they required the user to keep the palm in a horizontal position. For the abstract and iconic virtual hands, no tracking issues were detected.

The grabbing intent detection method relies on the angles of both metacarpal and proximal joints for all fingers, except the thumb. The thumb was not considered as we found no difference on the grasping quality and sometimes introduced noise. As shown on Figure 3, the contribution of metacarpal joints is less obvious than the one of the proximal joints. However, this feature was found to highly depend on users. Indeed, the grasping habit of everyone is different. To that extent, our choice was to consider both the metacarpal and the proximal joint angles. For grasping and release operations, a thresholding was done considering the sum of the orientation for each joint. Experimentally, we determined that the optimal grasping threshold was 290 degrees and the release threshold was 200 degrees. Finally, in order to provide additional feedback, the grabbed object changed its color when intersecting with the prehension area (green highlight) and when the object was grabbed (red highlight). 


\subsection{Apparatus and Participants}

Participants were immersed in the virtual environment using an Oculus Rift (DK2), in which head tracking was provided by the Oculus Rift and the participant's dominant hand was tracked using a Leap Motion. The physical setup (see Figure 4) was designed to ensure optimal tracking conditions for the Leap Motion. The Leap Motion was placed upside down, and anti-reflective tape was used on the shelf to limit infra-red interferences. In addition, the interaction space was constrained by the frame of the shelf. Participants were asked to only use their dominant hand when interacting with the virtual scene. Additionally, participants were asked to keep their other hand away from the field of view of the Leap motion to avoid detection artifacts. The virtual environment used in the experiment resembled the physical setup providing both a reference frame and passive haptic feedback when touching the bottom shelf. The application was developed using Unity and driven by a standard graphical workstation, which ensured a constant $75 \mathrm{~Hz}$.

Thirty-three male participants from inside and outside the lab took part in the experiment (aged from 21 to 44 years, $M=29.75$; $S D=9.67$ ). The population was restricted to male users as the realistic virtual hand was from a male avatar. Eighteen subjects did not have any previous experiment in virtual reality, seven had some previous experience and eight were familiar with VR. All participants except one were right-handed.

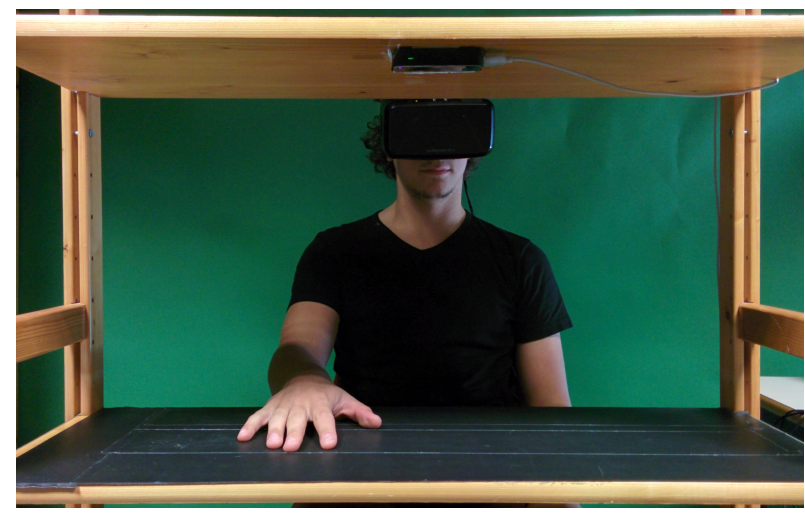

Figure 4: Experimental Setup. The interaction space was constrained by the shelf, which ensured an optimal tracking space for the Leap Motion (top). In addition, the bottom shelf provided passive haptic feedback.

\subsection{Experimental Protocol}

At the beginning of the experiment, participants, after signing the consent form, were briefed about the equipment and the experimental tasks. The experiment was subdivided into three blocks (one for each virtual hand representation) in which two different tasks were done, hereinafter referred as pick-and-place and spinning saw tasks (described below). Once the experimenter set up the VR equipment, the participant was able to explore the virtual environment by moving his head and to test the current virtual hand representation. After finishing each block, the experimenter removed the head-mounted display and participants were asked to fill a subjective questionnaire related to the corresponding virtual hand representation. Participants could take all the time they needed in order to continue the experiment. Participants took on average 10 minutes to perform each block and 45 minutes to finish the experiment.

\subsubsection{Pick-and-Place Task}

The pick-and-place task required participants to move a virtual cube from its original position towards a predefined position on the other side of the shelf, indicated by a red circle (see Figure 1 Left). The task had two goals in mind: first, to create a link between the real and the virtual hand through a repetitive interaction task, and second, to analyze the participant's behavior while avoiding potentially dangerous obstacles (see Figure 5). The sense of agency can be elicited by the sole means of controlling the virtual hand, while the potential hazardous situations can provide insights about the sense of ownership.

In order to avoid virtual objects popping in and out, a transition between each trial was introduced. Before each task, the user was asked to push a hand-shaped button in order to make a wooden curtain descend. When the curtain attained the shelf, the virtual elements were added to the virtual environment and the curtain automatically rose. This gave the feeling that the virtual scene elements were consistent, as they were not roughly appearing or disappearing. The system did not handle collisions between the virtual hand and the obstacles.
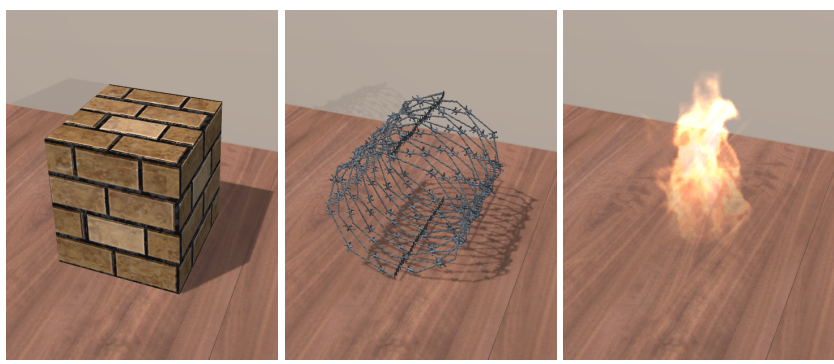

Figure 5: Virtual obstacles during the pick-and-place task. The brick is a tangible and non-threatening obstacle. The barbed wire is a tangible threatening object and the fire is intangible but threatening.

\subsubsection{Spinning Saw Task}

The spinning saw task was designed to assess the virtual hand control when actively performing a task that can potentially endanger the integrity of the virtual hand. The spinning saw task was performed just after the pick-and-place task, which ensured that participants became used to the virtual representation of their hand.

Participants had to place their hand in a specific location on the table, just besides a spinning saw (see Figure 1 Right). Participants had no time limit to perform the task, and the only instruction provided was to place the virtual hand on the designated mark. This task aimed to study how a danger in the virtual environment is perceived, and whether participants will risk the integrity of their virtual hand. The participant's behavior provides insights about the sense of ownership.

\subsection{Design and Hypotheses}

The pick-and-place task followed a full factorial $3 \times 4 \times 2$ design: virtual hand representation ( 3 levels: abstract, iconic and realistic), obstacle (4: levels, none, brick, wire and fire) and direction of the movement (2 levels: right-to-left and left-to-right). All variables were within-subjects. The experiment was divided into three blocks, one for each virtual hand representation. To minimize the ordering effects, the virtual hand condition was counterbalanced (Latin-square design) while obstacle and direction conditions were fully randomized. For each combination, participants did five repetitions, resulting in a total of 120 pick-and-place tasks. Ten additional training trials without any obstacles were included at the beginning of each block, thus ensuring that participants understood the task and the hand control. The main dependent variables were the task completion time (s) and the placement precision $(\mathrm{cm})$, the 


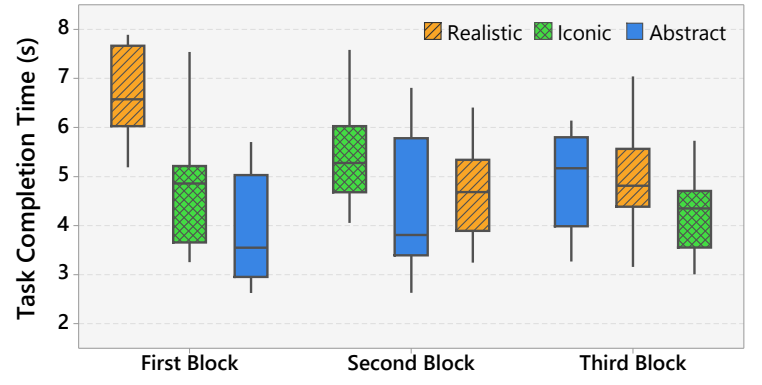

Figure 6: Boxplot of the task completion time considering the virtual hand representation and the ordering.

latter being measured by computing the distance between the object center and the target location (XZ plane). Additionally, we measured the grasping thrust (sum of metacarpal and proximal join angles) and the elevation at the midpoint of the trajectory.

The spinning saw task only had one within-subjects independent variable, the virtual hand representation. This task was the last trial performed in each block (after the pick-and-place task). Participants only performed the spinning saw task once for each representation, to decrease the habituation effect. We measured the time (s) required to place the hand in the designed location. The task was considered finished when any part of the virtual hand touched the placement mark.

In addition to the quantitative measures, participants were asked to assess their subjective impressions after each block by the means of subjective questionnaires (see Table 1). The questionnaire was mainly inspired by the works of Botvinick et Cohen [5] and Longo et al. [19] Finally, we recorded the described trajectories in order to analyze changes in participants' behavior during both tasks. According to our experimental design, our main hypotheses were:

H1 Faster manipulation time for simplified virtual hands.

H2 Increased manipulation precision for simplified virtual hands.

H3 Increased placement time for the realistic virtual hand.

H4 Increased collision avoidance for the realistic virtual hand.

H5 Increased sense of agency for the realistic virtual hand.

H6 Increased sense of ownership for the realistic virtual hand.

\subsection{Analysis}

Parametric data was analyzed using factorial ANOVA analysis. Tukey pairwise tests $(\alpha=0.05)$ were done when needed, only significant differences are discussed $(p<0.05)$. When ordering effects were observed, the order was included as a between-subjects factor.

\subsubsection{Pick-and-Place Task}

The four way ANOVA, virtual hand, obstacle, direction and order vs task completion time showed three relevant significant effects. First, there was a main effect of obstacle $(F(3,90)=54.13$; $\left.p<0.001 ; \eta_{p}^{2}=0.64\right)$. Post-hoc tests showed that users required significantly more time to perform the task during the fire condition $(M=5.51 s ; S D=1.86 s)$, followed by the brick $(M=4.9 ; S D=1.53)$ and barbed wire $(M=4.9 s ; S D=1.53 s)$ conditions. The condition without obstacle resulted in the fastest task completion times $(M=4.25 \mathrm{~s}$; $S D=1.35 s)$. Second, there was a two-way interaction effect between order and virtual hand $\left(F(4,60)=24.52 ; p<0.001 ; \eta_{p}^{2}=0.61\right)$ (see Figure 6). Post-hoc tests showed that there was a significant increase in performance between the first and the third block for the realistic virtual hand. In contrast, between the second and the third block all three techniques achieved comparable performances. Third, there was a main effect of direction $(F(1,30)=395.12$; $\left.p<0.001 ; \eta_{p}^{2}=0.92\right)$, post-hoc tests showed higher task completion times for the left-to-right trials $(M=5.42 s ; S D=1.64 s)$ than the rightto-left trials $(M=4.37 s ; S D=1.47 s)$. This effect was mainly due to the fact that we considered the time before the grasping. Users therefore had a longer distance to cover before grasping the object for the left-to-right condition which consistently increased the task completion time.

The four way ANOVA, virtual hand, obstacle, direction and order vs placement precision showed two relevant significant effects. First, there was a strong main effect of virtual hand representation $\left(F(2,60)=26.24 ; p<0.001 ; \eta_{p}^{2}=0.46\right)$. Post-hoc tests showed that participants were significantly less accurate with the realistic hand $(M=3.52 \mathrm{~cm} ; S D=1.34 \mathrm{~cm})$ compared to both the iconic $(M=2.7 \mathrm{~cm} ; S D=1.17 \mathrm{~cm})$ and the abstract $(M=2.70 \mathrm{~cm} ; S D=0.99 \mathrm{~cm})$ representations. Second, there was a moderate effect on the obstacle $\left(F(3,90)=6.30 ; p<0.001 ; \eta_{p}^{2}=0.17\right)$. Post-hoc tests showed that the accuracy for the fire condition was the lowest $(M=3.27 \mathrm{~cm}$; $S D=0.94 \mathrm{~cm}$. Nevertheless, the differences among conditions are lower than $1 \mathrm{~cm}$.

Regarding the mean grasping thrust, as no significant differences were found in terms of distance, order and block, data was pooled and only the virtual hand representation was considered as a factor. The one-way ANOVA virtual hand representation vs grasping thrust showed a significant effect on virtual hand representation $\left(F(2,64)=4.9 ; p<0.05 ; \eta_{p}^{2}=0.13\right)$ Post-hoc tests showed that the grasp thrust for the realistic virtual hand $(M=460.64 ; S D=52.04)$ was significantly lower than the grasp thrust for the abstract virtual hand $(M=489.17 ; S D=55.27)$.

Finally, we explored the hand trajectory when performing the pick-and-place task. In order to characterize the trajectories, we considered the elevation at the midpoint of the trajectory as the main feature. We only found a significant effect on obstacle $\left(F(3,96)=55.84 ; p<0.01 ; \eta_{p}^{2}=0.64\right)$. Post-hoc tests only showed that the mean elevation was lower only for the non-obstacle condition $(M=10.23 \mathrm{~cm} ; S D=3.6 \mathrm{~cm})$ compared to the other conditions $(M=17.5 \mathrm{~cm} ; S D=6.64 \mathrm{~cm})$. Nevertheless, we observed higher variability in the avoidance strategies when avoiding the fire. Figure 8 shows the mean trajectory for each participant. We can observe that different strategies were used to avoid the obstacle, such as avoiding the fire sideways. Another observation from the trajectories is that few participants did not avoid the obstacles, even for the solid ones. No correlation was found regarding their VR experience.

\subsubsection{Spinning Saw Task}

The two-way ANOVA virtual hand representation and order vs task completion time showed a main effect of virtual hand representation $\left(F(2,81)=4.22 ; p<0.05 ; \eta_{p}^{2}=0.09\right)$ and of order $\left(F(2,81)=6.57 ; p<0.005 ; \eta_{p}^{2}=0.14\right)$. Tukey post-hoc tests showed that participants took significantly more time to perform the task using the realistic virtual hand $(M=3.21 ; S D=1.15)$ than the abstract virtual hand $(M=2.46 ; S D=0.78)$. Moreover, participants took significantly more time to perform the task the first time $(M=3.34$; $S D=1.06)$ in comparison with both the second $(M=2.61 ; S D=0.77)$ and the third $(M=2.58 ; S D=0.96)$ block. No interaction effect was found.

We also analyzed the final position of the participants forearm. Figure 7 shows that there were three main strategies to perform the task, approaching from the top (white) from the side (green) or from the front (red). We observed a tendency of increased saw avoidance for the realistic hand condition ( 9 collisions) compared to the abstract (14) or the iconic (13) virtual hand. 

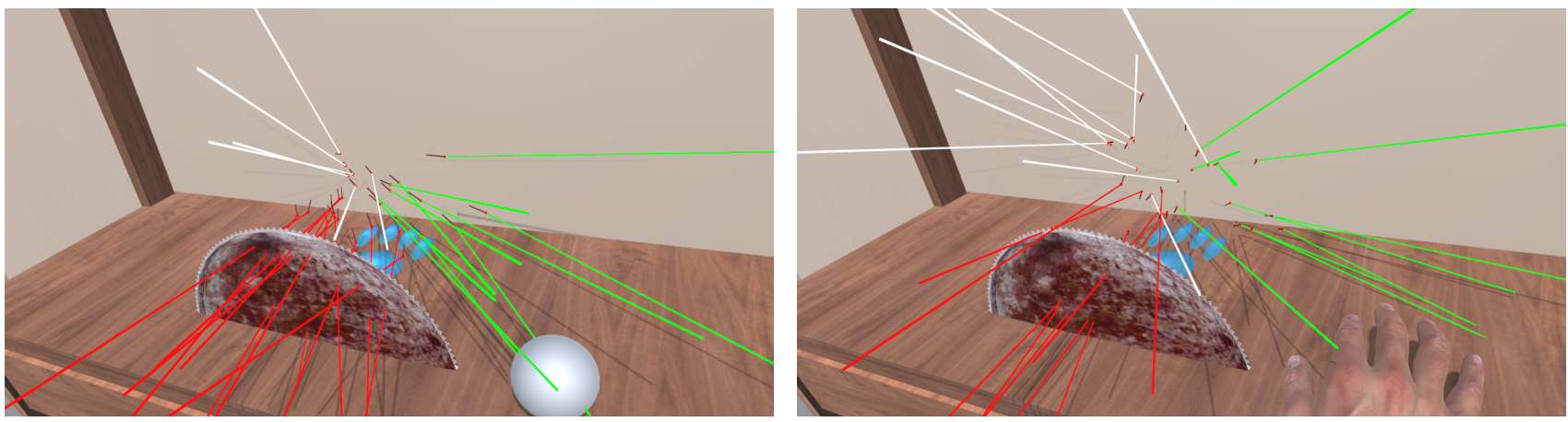

Figure 7: Final position and orientation of the forearm during the spinning saw task for the abstract (left) and realistic (right) conditions. The color determines whether the forearm intersected with the saw (red), the user approached from the top (white) or from the side (green).
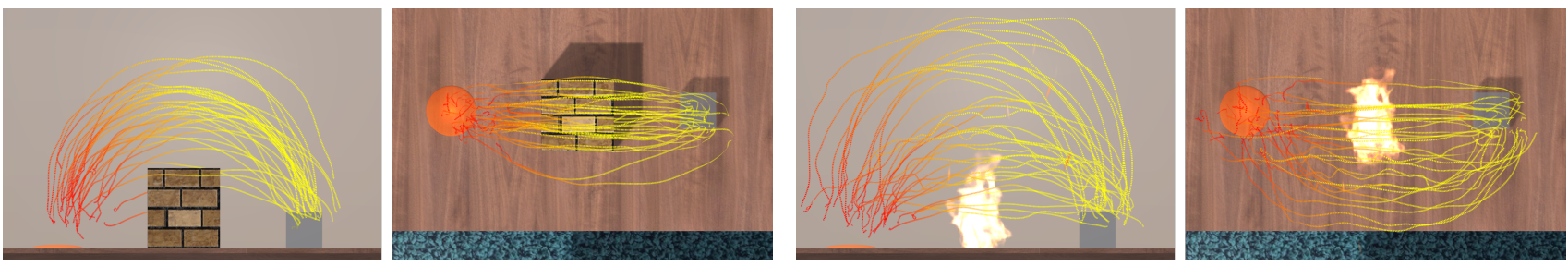

Figure 8: Mean trajectories when avoiding the brick (left) and the fire (right) obstacles for the realistic virtual hand. The fire increased the trajectory variability as participants showed different avoidance strategies.

\subsubsection{Questionnaires}

The data from the questionnaires was analyzed using the Friedman rank test and Wilcoxon pairwise test. The questionnaire is split into agency- (A1-A6) and ownership-related (O1-O8) questions. Before performing the analysis, ordering effects were tested, but no significant ordering effects were found. Table 1 provides the summary of the results.

According to the agency results (A1-A6), we observe that participants perceived that the realistic virtual hand was more difficult to control (A1), as they expected the virtual hand to precisely follow their movements (A2). This is also supported by the fact that participants considered that performing the different tasks using the realistic virtual hand was slightly more difficult (A3 and A4). Nevertheless, all virtual hand representations elicited a strong sense of agency, which shows that participants had the feeling of controlling all three virtual representations of the virtual hand.

Regarding the ownership-related questions (O1-O8), we observe higher ratings for the realistic virtual hand in terms of the virtual hand being part of their body $(\mathrm{O} 1)$, the coupling between the real and the virtual hand $(\mathrm{O} 3)$ and increased feeling of danger towards the virtual hand $(\mathrm{O} 4)$ and their real hand (O5). The control question $\mathrm{O} 2$ showed no significant contradictory effects. There was no significant differences in participants' behavior to avoid obstacles (O6) although they considered that the virtual hand was able to go through virtual obstacles (O8). Overall, we can state that the sense of ownership was higher for the realistic virtual hand.

\subsection{Discussion}

The results from the pick-and-place task showed that simplified virtual hands provide faster and more accurate interactions supporting hypotheses [H1] and [H2]. However, the observed ordering effects show that the differences are more visible during the first block of the experiment. As long as the experiment advances the differences among them decrease. This decrease in performance can be explained by the lack of feedback. The realistic virtual hand occluded the virtual object during grasping and release operations, and no haptic feedback was provided. This effect was stronger in the first block as participants were still not adapted to the grasping/release control. In addition, the sense of agency was higher for the abstract and the iconic virtual hands in A1 (I felt as if the virtual representation of the hand moved just like I wanted it to), A3 (Perceived task difficulty) and A4 (I felt like I was able to interact with the environment the way I wanted to). This decrease of the agency towards the realistic virtual hand can be correlated with its decreased performance. We can hypothesize that increased task completion times indicate a decreased motor control which can be perceived for the user as a decrease in the sense of agency. A second explanation is the limitation of the tracking system. Although it did not happened frequently, in some cases, the Leap Motion mistook the participant's right hand for the left one, providing strange virtual finger configurations. Interestingly, the reaction of participants when there was a difference between their physical hand and the virtual one was to shake their hand, as if they tried to "shuffle it back". This could be one of the reasons of the lower scores for question $\mathrm{A} 1$ and higher scores for question $\mathrm{O} 3$ (I felt that I was losing the control of my hand when the virtual hand was not responding properly). Either way, these results do not support [H5].

In contrast, we did not observe any significant difference among virtual hand representations in the collision avoidance strategy, which does not support hypothesis [H4]. On the vast majority of cases, although the natural behavior was to avoid obstacles, the avoidance behavior did not depend on the virtual hand representation. This is also supported by the subjective questionnaires (O6aO6c) in which participants reported that they kept a similar strategy for each virtual hand representation. Interestingly, participants tended to avoid the fire obstacle even in iconic and abstract conditions. Some participants even had difficulties passing above the fire, which can be interpreted as an extension of the volume of the danger zone of the fire outside of its visible area, being supported by alternative avoiding strategies like going around it (see Figure 8). On the other hand, two participants systematically went through all virtual obstacles. 
Table 1: Statistical summary for the questionnaire responses (7-Likert scale). Friedman rank tests and Wilcoxon post-hoc tests were used. Mean and standard deviations not sharing a subindex $\left({ }^{1}\right.$ or $\left.{ }^{2}\right)$ indicate significant differences $(\alpha=0.05)$. The mean and standard deviation for all virtual hand conditions is provided when no significant differences were found.

\begin{tabular}{|c|c|c|c|c|}
\hline ID & Question & $\operatorname{Abstract}(\bar{x}, \sigma)$ & Iconic $(\bar{x}, \sigma)$ & Realistic $(\bar{x}, \sigma)$ \\
\hline A1 & $\begin{array}{l}\text { I felt as if the virtual representation of the hand moved just like I wanted it to, as if it was } \\
\text { obeying my will. }\end{array}$ & $(5.70,1.05)^{2}$ & $(5.36,0.99)^{2}$ & $(4.49,1.40)^{1}$ \\
\hline A2 & I expected the virtual representation of the hand to react in the same way as my own hand. & $(4.85,1.60)^{1}$ & $(5.18,1.29)^{1}$ & $(6.09,1.07)^{2}$ \\
\hline A3 & The task was ( 1 difficult, 7 easy) to perform. & $(6.33,0.84)^{2}$ & $(6.12,0.82)^{1,2}$ & $(5.67,1.24)^{1}$ \\
\hline A4 & I felt like I was able to interact with the environment the way I wanted to. & $(5.79,1.17)^{2}$ & $(5.61,0.90)^{2}$ & $(4.67,1.11)^{1}$ \\
\hline A5 & I felt that the interaction with the environment was realistic. & \multicolumn{3}{|c|}{$(5.12,1.63)$} \\
\hline A6 & $\begin{array}{l}\text { I felt like I controlled the virtual representation of the hand as if it was part of my own } \\
\text { body. }\end{array}$ & \multicolumn{3}{|c|}{$(4.79,1.32)$} \\
\hline $\mathrm{O} 1$ & I felt as if the virtual representation of the hand was part of my body. & $(3.97,1.50)^{1}$ & $(4.52,1.37)^{1}$ & $(5.55,1.06)^{2}$ \\
\hline $\mathrm{O} 2$ & I felt as if the virtual representation of the hand was someone else's. & \multicolumn{3}{|c|}{$(2.21,1.46)$} \\
\hline $\mathrm{O} 3$ & $\begin{array}{l}\text { I felt that I was losing the control of my hand when the virtual hand was not responding } \\
\text { properly. }\end{array}$ & $(2.46,1.77)^{1}$ & $(3.76,2.05)^{1}$ & $(4.33,2.14)^{2}$ \\
\hline $\mathrm{O} 4$ & I thought that the virtual representation of the hand could be harmed by the virtual danger. & $(3.66,1.94)^{1}$ & $(3.88,1.82)^{1}$ & $(4.91,1.89)^{2}$ \\
\hline O5 & I felt that my real body was endangered during the experiment. & $(1.94,1.46)^{1}$ & $(2.24,1.58)^{1,2}$ & $(3.00,1.95)^{2}$ \\
\hline O6a & Did you try to avoid the virtual obstacle (fire) while performing the task? & \multicolumn{3}{|c|}{$(5.99,1.70)$} \\
\hline O6b & Did you try to avoid the virtual obstacle (brick) while performing the task? & \multicolumn{3}{|c|}{$(6.04,1.77)$} \\
\hline O6c & Did you try to avoid the virtual obstacle (barbed wire) while performing the task? & \multicolumn{3}{|c|}{$(5.90,1.94)$} \\
\hline $\mathrm{O} 7$ & $\begin{array}{l}\text { I felt my own hand tickling whenever the virtual representation of the hand went through } \\
\text { a virtual obstacle. }\end{array}$ & \multicolumn{3}{|c|}{$(2.19,1.49)$} \\
\hline O8 & I felt that the virtual representation of the hand was able to go through the virtual obstacles. & \multicolumn{3}{|c|}{$(4.31,1.95)$} \\
\hline
\end{tabular}

The task completion time during the spinning saw task showed that participants required significantly more time to finish the task using the realistic virtual hand, thus supporting [H3]. Figure 7 shows that participants had the tendency to avoid the saw to be collocated with their forearm, with a stronger effect for the realistic virtual hand representation, which supports [H6]. From these results, we can hypothesize that participants were more careful when performing the task due to the increased feeling of danger. This is supported by the results from the subjective questionnaires in which participants ranked the potential virtual and real danger higher for the realistic virtual hand (O4-O5). Furthermore, different participant profiles were observed. For instance, the body ownership component of a user was particularly strong as he hesitated to put his hand behind the circular saw for quite a long time with the realistic hand condition. In contrast, a few participants went through all obstacles, and even tried to put their virtual hand in the saw, which shows a lower ownership component.

\subsection{Limitations}

Bare hand interactions present tracking and interaction challenges and no perfect solution exists yet. Our choice was to use depth sensing technology to avoid the user having to wear bulky equipment and to provide an interaction as natural as possible. However, depth sensing technologies are prone to occlusions and noisy reconstructions which could have biased the perception of participants especially for the realistic condition. Nevertheless, although tracking artifacts sometimes appeared, the experimental tasks were designed to minimize such artifacts. Participants were able to perform the tasks efficiently and the measured levels of agency and ownership show a high degree of virtual embodiment.

One potential source of bias, especially during the spinning saw task, could have been the fact that the realistic virtual hand provided visual feedback for the forearm. Participants could have just avoided interpenetration between their forearm and the spinning saw. Adding a virtual forearm was our choice as we considered that a realistic virtual hand floating in mid-air would feel strange. Still, we observed non-expected behaviors: some participants avoided the saw when no forearm was displayed and some participants did not avoid the saw when the forearm was displayed. This result shows that the role of the forearm could not only be related to the visual feedback, but also the proprioception of the real forearm. Additional studies are required to assess this question.

\section{Global Discussion}

From the results obtained in the experiment, we can now partially answer the two main research questions. Does the virtual representation of the hand alter the sense of agency? Participants' subjective impressions showed that the sense of agency was influenced by the virtual hand representation, with the abstract and iconic representations being the ones providing the highest sense of agency. This was an unexpected result as we hypothesized that the increased number of degrees of freedom of the virtual hand animation would have elicited a stronger sense of agency. The decreased performance for the realistic virtual hand and the finger tracking limitations could have influenced the results. In both cases, there is a reduction of the perceived control when manipulating and interacting with the virtual hand. Although, all virtual hand representations elicited a strong sense of agency, more accurate finger tracking and improved sensory feedback could result in a stronger sense of agency.

Does the virtual representation of the hand alter the sense of ownership? Both qualitative and quantitative data suggest that the realistic virtual hand representation elicits stronger sense of ownership. Interestingly, although the sense of agency is also an important factor for the sense of ownership, we observe that the decreased sense of agency observed for the realistic virtual hand was not enough to alter the sense of ownership. 


\section{CONCLUSION}

The presented experiment explored the effects of the virtual hand representation on the senses of agency and ownership. On the one hand, we have observed that the sense of agency does not seem to be related to the amount of degrees of freedom the user can control, but rather on their efficiency to control them. Even the abstract virtual hand which required only 3 degrees of freedom provided a high sense of agency. This suggests that the design of the user's avatar has to take into account the quality of the tracking system in order to provide a seamless control. Furthermore, as it has been acknowledged in the literature, there is no specific need to provide realistic avatar representation to increase the sense of agency. On the other hand, the sense of ownership is dependent on the virtual representation of the virtual hand and the need of morphological resemblance is required to increase the sense of ownership. Interestingly, this result still holds even when the sense of agency for the realistic virtual hand was lower, thus suggesting that the provided level of control was sufficient to ensure an ownership illusion. Nevertheless, additional studies are required to explore the link between agency and ownership in interactive VR scenarios.

\section{ACKNOWLEDGEMENTS}

The authors wish to thank all the people who make this work possible: Julian Joseph for his valuable help in the design of the experiment, Adrien Girard for his fruitful discussions and all the people participating in the experiments.

\section{REFERENCES}

[1] O. Blanke. Multisensory brain mechanisms of bodily selfconsciousness. Nature Reviews Neuroscience, 13:556-571, 2012.

[2] O. Blanke and T. Metzinger. Full-body illusions and minimal phenomenal selfhood. Trends in cognitive sciences, 13(1):7-13, 2009.

[3] D. Borland. Integrating head and full-body tracking for embodiment in virtual characters. In IEEE Virtual Reality, pages 81-82, 2013.

[4] C. Borst and A. Indugula. Realistic virtual grasping. In IEEE Virtual Reality, pages 91-320, 2005

[5] M. Botvinick and J. Cohen. Rubber hands 'feel' touch that eyes see. Nature, 391(6669):756, 1998.

[6] E. A. Caspar, A. Cleeremans, and P. Haggard. The relationship between human agency and embodiment. Consciousness and Cognition, 33:226-36, 2015.

[7] D. M. Clower and D. Boussaoud. Selective use of perceptual recalibration versus visuomotor skill acquisition. Journal of Neurophysiology, 84(5):2703-8, 2000.

[8] F. De Vignemont. Embodiment, ownership and disownership. Consciousness and Cognition, 20(1):82-93, 2011.

[9] H. Ehrsson. How many arms make a pair? Perceptual illusion of having an additional limb. Perception, 38(2):310-312, 2009.

[10] D. Friedman, R. Pizarro, K. Or-Berkers, S. Neyret, X. Pan, and M. Slater. A method for generating an illusion of backwards time travel using immersive virtual reality-an exploratory study. Frontiers in Psychology, 5:943, 2014.

[11] M. Gonzalez-Franco, D. Perez-Marcos, B. Spanlang, and M. Slater. The contribution of real-time mirror reflections of motor actions on virtual body ownership in an immersive virtual environment. In IEEE Virtual Reality, pages 111-114, 2010.

[12] A. Guterstam, Z. Abdulkarim, and H. H. Ehrsson. Illusory ownership of an invisible body reduces autonomic and subjective social anxiety responses. Scientific reports, 5:9831, 2015.

[13] A. Kalckert and H. H. Ehrsson. The moving rubber hand illusion revisited: Comparing movements and visuotactile stimulation to induce illusory ownership. Consciousness and Cognition, 26(1):117132, 2014.

[14] K. Kilteni, I. Bergstrom, and M. Slater. Drumming in immersive virtual reality: the body shapes the way we play. IEEE Transactions on Visualization and Computer Graphics, 19(4):597-605, 2013.
[15] K. Kilteni, R. Groten, and M. Slater. The Sense of Embodiment in Virtual Reality. Presence: Teleoperators and Virtual Environments, 21:373-387, 2012.

[16] K. Kilteni, J. M. Normand, M. V. Sanchez-Vives, and M. Slater. Extending body space in immersive virtual reality: A very long arm illusion. PLoS ONE, 7(7), 2012.

[17] P. G. Kry, A. Pihuit, A. Bernhardt, and M.-P. Cani. HandNavigator: Hands-on Interaction for Desktop Virtual Reality. In ACM symposium on Virtual Reality Software and Technology, pages 53-60, 2008.

[18] B. Lenggenhager, T. Tadi, T. Metzinger, and O. Blanke. Video ergo sum: manipulating bodily self-consciousness. Science, 317(5841):1096-1099, 2007.

[19] M. R. Longo, F. Schüür, M. P. M. Kammers, M. Tsakiris, and P. Haggard. What is embodiment? A psychometric approach. Cognition, 107(3):978-98, 2008.

[20] J.-L. Lugrin, J. Latt, and M. E. Latoschik. Avatar Anthropomorphism and Illusion of Body Ownership in VR. In IEEE Virtual Reality (Posters), 2015.

[21] M. Moehring and B. Froehlich. Effective manipulation of virtual objects within arm's reach. In IEEE Virtual Reality, p. 131-138, 2011.

[22] T. C. Peck, S. Seinfeld, S. M. Aglioti, and M. Slater. Putting yourself in the skin of a black avatar reduces implicit racial bias. Consciousness and Cognition, 22(3):779-787, 2013.

[23] V. I. Petkova and H. H. Ehrsson. If I were you: Perceptual illusion of body swapping. PLoS ONE, 3(12), 2008.

[24] V. I. Petkova and H. H. Ehrsson. When right feels left: Referral of touch and ownership between the hands. PLOS ONE, 4(9), 2009.

[25] V. I. Petkova, M. Khoshnevis, and H. H. Ehrsson. The perspective matters! Multisensory integration in egocentric reference frames determines full-body ownership. Frontiers in Psychology, 2:35, 2011.

[26] I. V. Piryankova, H. Y. Wong, S. A. Linkenauger, C. Stinson, M. R. Longo, H. H. Bülthoff, and B. J. Mohler. Owning an overweight or underweight body: distinguishing the physical, experienced and virtual body. PLoS ONE, 9(8), 2014.

[27] I. Poupyrev, T. Ichikawa, S. Weghorst, and M. Billinghurst. Egocentric Object Manipulation in Virtual Environments: Empirical Evaluation of Interaction Techniques. Comp. Graph. Forum, 17(3):41-52, 1998.

[28] A. Pusch, O. Martin, and S. Coquillart. Effects of hand feedback fidelity on near space pointing performance and user acceptance. In IEEE International Symposium on VR Innovation, p. 97-102, 2011.

[29] M. V. Sanchez-Vives, B. Spanlang, A. Frisoli, M. Bergamasco, and M. Slater. Virtual hand illusion induced by visuomotor correlations. PLOS ONE, 5(4), 2010.

[30] M. Slater, D. Perez-Marcos, H. H. Ehrsson, and M. V. Sanchez-Vives. Towards a digital body: the virtual arm illusion. Frontiers in Human Neuroscience, 2:6, 2008.

[31] M. Slater, B. Spanlang, M. V. Sanchez-Vives, and O. Blanke. First person experience of body transfer in VR. PLOS ONE, 5(5), 2010.

[32] J. Sreng, A. Lécuyer, C. Mégard, and C. Andriot. Using visual cues of contact to improve interactive manipulation of virtual objects in industrial assembly/maintenance simulations. IEEE Transactions on Visualization and Computer Graphics, 12(5):1013-20, 2006.

[33] W. Steptoe, A. Steed, and M. Slater. Human tails: ownership and control of extended humanoid avatars. IEEE Transactions on Visualization and Computer Graphics, 19(4):583-90, 2013.

[34] M. Tsakiris and P. Haggard. The rubber hand illusion revisited: visuotactile integration and self-attribution. Journal of Experimental Psychology. Human Perception and Performance, 31(1):80-91, 2005.

[35] M. Tsakiris, G. Prabhu, and P. Haggard. Having a body versus moving your body: How agency structures body-ownership. Consciousness and Cognition, 15(2):423-32, 2006.

[36] T. Ullmann and J. Sauer. Intuitive virtual grasping for non haptic environments. In IEEE Pacific Conference on Computer Graphics and Applications, pages 373-457, 2000.

[37] A. C. Wellerdiek, M. Leyrer, E. Volkova, D.-S. Chang, and B. Mohler. Recognizing your own motions on virtual avatars. In ACM symposium on Applied Perception, page 138, 2013.

[38] A. S. Won, J. Bailenson, J. Lee, and J. Lanier. Homuncular Flexibility in Virtual Reality. Journal of Computer-Mediated Communication, 20(3):241-259, 2015. 